\title{
ONE-DIMENSIONAL GAME OF LIFE AND ITS GROWTH FUNCTIONS
}

\author{
MOHAMMAD H. AHMADI \\ Drpartment of Mathemat.jcs and computer Science \\ University of Wisconsin \\ Whitewater, WI 5.3190 \\ (Received June 6, 1990 and in revised form October 28, 1991)
}

AHSTRAC'T. We start with finitely many 1 's and possibly some 0 's in between. Then each entry in the other rows is obtained from the Base 2 sum of the two numbers diagonally above it in the preceding rov. We may formulate the game as follows:

Define $d_{1, j}$ recursively for 1 , a non-negative integer, and $j$ an arbitrary integer by the rules:

$$
\begin{aligned}
d_{0, j} & =\left\{\begin{array}{l}
1 \\
0 \text { or } 1 \text { for } j=0, k \\
\text { for } 0<j<k
\end{array}\right. \\
d_{0, j} & =0 \text { for } j<0 \text { or } j>k \\
d_{i+1, j} & =d_{i, j+1}(\bmod 2) \text { for } i \geq 0 .
\end{aligned}
$$

Nov, if ve interpret the number of 1 's in row $i$ as the coefficient $a_{i}$ of a formal power series, then ve obtain a grovth function,

$f(x)=\sum_{i=0}^{\infty} a_{i} x^{i}$. It is interesting that there are cases for which this grovth

function factors into an infinite product of polynomials. Furthermore, ve shall show that this pover series never represents a rational function.

1991 AMS SUBJECT CLASSIFICATION CODES: 05A10

KEYWORDS AND PHRASES: Game of Life, Growth Functions

1. INTRODUCTION

To explain in vords:

(I) describes the starting configuration with finitely many 1 's, and possibly some 0 's in betveen. (Picture this as row zero).

(II) says that all entries on both sides of the starting configuration are zero. (Note that considering the 0 's on both sides contributes nothing).

(III) says that each entry (in the other rovs) is obtained from the Base 2 sum of the two numbers diagonally above it in the preceding rov.

Now, if ve interpret the number of 1 's in rov $i$ as the coefficient $a_{i}$ of a formal pover series, then ve obtain a growth function:

$f(x)=\sum_{i=0}^{\infty} a_{i} x^{i}$. It is interesting that there are cases for wich this grovth 
function factors into an infinite product of polynomials. But there are cases in which the pattern is less regular. Nevertheless, ve shall shov the folloving.

THEOREM. In a One-dimensional Game of Life, no matter what the starting configuration is, (finitely many 1 's and possibly some 0 's in betreen), the associated grovth function never represents a rational function.

First ve look at some examples:

EXAMPLE 1. Suppose the starting configuration has one 1 . We get the folloving configuration called "Fundamental Configuration" throughout this paper.

Rov Term

\begin{tabular}{|c|c|c|}
\hline 0 & 1 & 1 \\
\hline 1 & $2 x$ & 101 \\
\hline 2 & $2 x^{2}$ & 100001 \\
\hline 3 & $4 x^{3}$ & 1010101 \\
\hline 4 & $2 x^{4}$ & 10000000001 \\
\hline 5 & $4 x^{5}$ & 1010000000101 \\
\hline 6 & $4 x^{6}$ & 1000100010001 \\
\hline
\end{tabular}

The resulting grovth function is:

$$
f(x)=\sum_{i=0}^{\infty} a_{i} x^{i}=1+2 x+2 x^{2}+4 x^{3}+2 x^{4}+4 x^{5}+4 x^{6}+\cdots \cdot
$$

We observe that the configuration above is essentially the configuration obtained by reducing modulo 2 each element of Pascal's triangle of binomial coefficients. To obtain the reduced Pascal's triangle from configuration in example 1 simply remove every second entry of each row beginning with the entry 0 following the initial 1 of that rov. Each entry removed will be a zero.

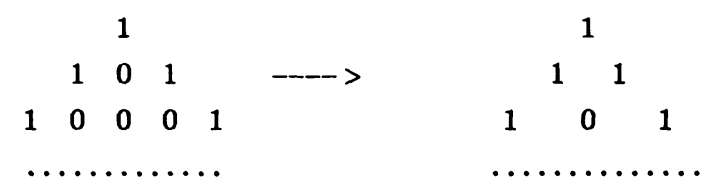

This connection with Pascal's triangle can be used to explain the fact that, for each $\mathrm{j} \geq 1$, row $2^{\mathrm{j}}$ has precisely two non-zero entries, one at each end. In other vords, for every integer $j \geq 1$, rov $2^{j}$ has two copies of starting configuration, one at each end. Therefore, $a_{2} j=2 a_{0}$.

Worthy of notice is that Wolfram [3] has shown: In Pascal's Triangle of binomial coefficients reduced modulo 2 , when infinitely many rovs are included, the limiting pattern exhibits a fractal self-similarity and is characterized by a "fractal dimension" $\log _{2} 3$.

DEFINITION 1. For any integer $j \geq 1$, the set of rovs between rov $2^{j-1}$ and $2^{j}-1$ inclusive, is called the jth Stage. Note that rov $2^{j}, j \geq 1$, is the initial row of the $j$ th Stage. Stage 0 , is the 0 th rov or the initial rov of the
configuration. 
EXAMPLE 2. If $d_{0, j}=1, j=0,1$ then ve get the follawing configuration: Row T'erm

\begin{tabular}{|c|c|c|c|}
\hline 0 & 2 & 11 & $<-$ stage 0 \\
\hline 1 & $4 \mathrm{x}$ & $\begin{array}{llll}1 & 1 & 1 & 1\end{array}$ & $<-$ stage 1 \\
\hline 2 & $4 x^{2}$ & 11100011 & \\
\hline 3 & $8 x^{3}$ & $\begin{array}{lllllllll}1 & 1 & 1 & 1 & 1 & 1 & 1 & 1\end{array}$ & $<--$ stage 2 \\
\hline 4 & $4 x^{4}$ & 110000000011 & \\
\hline 5 & $8 x^{5}$ & $\begin{array}{lllllllllllll}1 & 1 & 1 & 1 & 0 & 0 & 0 & 0 & 1 & 1 & 1 & 1\end{array}$ & \\
\hline 6 & $8 x^{6}$ & $\begin{array}{llllllllllllll}1 & 1 & 0 & 0 & 1 & 1 & 0 & 0 & 1 & 1 & 0 & 0 & 1 & 1\end{array}$ & \\
\hline 7 & $16 x^{7}$ & $\begin{array}{llllllllllllllll}1 & 1 & 1 & 1 & 1 & 1 & 1 & 1 & 1 & 1 & 1 & 1 & 1 & 1 & 1 & 1\end{array}$ & \\
\hline 8 & $4 x^{8}$ & 11000000000000000000001 & \\
\hline
\end{tabular}

The resulting grovth function $\mathbf{g}(\mathbf{x})$ may be written as:

$$
g(x)=2+4 x+4 x^{2}+8 x^{3}+4 x^{4}+8 x^{5}+8 x^{6}+16 x^{7}+4 x^{8}+\cdots
$$

EXAMPLE 3. Let $d_{0, j}=1$ for $j=0,1,2$. This yields the following configuration:

Rov

$$
0
$$$$
1
$$

2

3

4

5

6

7

8
Term

3

$4 \mathrm{x}$

$6 \mathrm{x}^{2}$

$6 \mathrm{x}^{3}$

$6 \mathrm{x}^{4}$

$8 \mathrm{x}^{5}$

$12 \mathrm{x}^{6}$

$10 x^{7}$

$6 x^{8}$
111

$\begin{array}{lllll}1 & 1 & 0 & 1 & 1\end{array}$

11110111

11010101011

$\begin{array}{lllllllllll}11 & 1 & 0 & 0 & 0 & 0 & 0 & 1 & 1 & 1\end{array}$

1101100011011

1110111011110111

11010101010101011

111000000000000000111

The grovth function $h(x)$ is:

$$
h(x)=3+4 x+6 x^{2}+6 x^{3}+6 x^{4}+8 x^{5}+12 x^{6}+10 x^{7}+6 x^{8}+\cdots
$$

FACTORIZATION OF THE FUNDAMENTAL GRONTH FUNCTION. If we look at the configurations in examples 1 - 3 carefully, ve observe that there is in each a recurrent triangular pattern. This is dramatically illustrated in the more extensive computer printouts of figures $1-4$, reproduced below. Note that in these figures the zeros are not printed.

In fundamental configuration (Fig. 1) since in each Stage ve have two copies of the preceding triangle (or Stage), hence ve may factor its grovth function, $f(x)$, as follovs:

$$
f(x)=(1+2 x)\left(1+2 x^{2}\right)\left(1+2 x^{4}\right)\left(1+2 x^{8}\right)\left(1+2 x^{12}\right) \ldots
$$




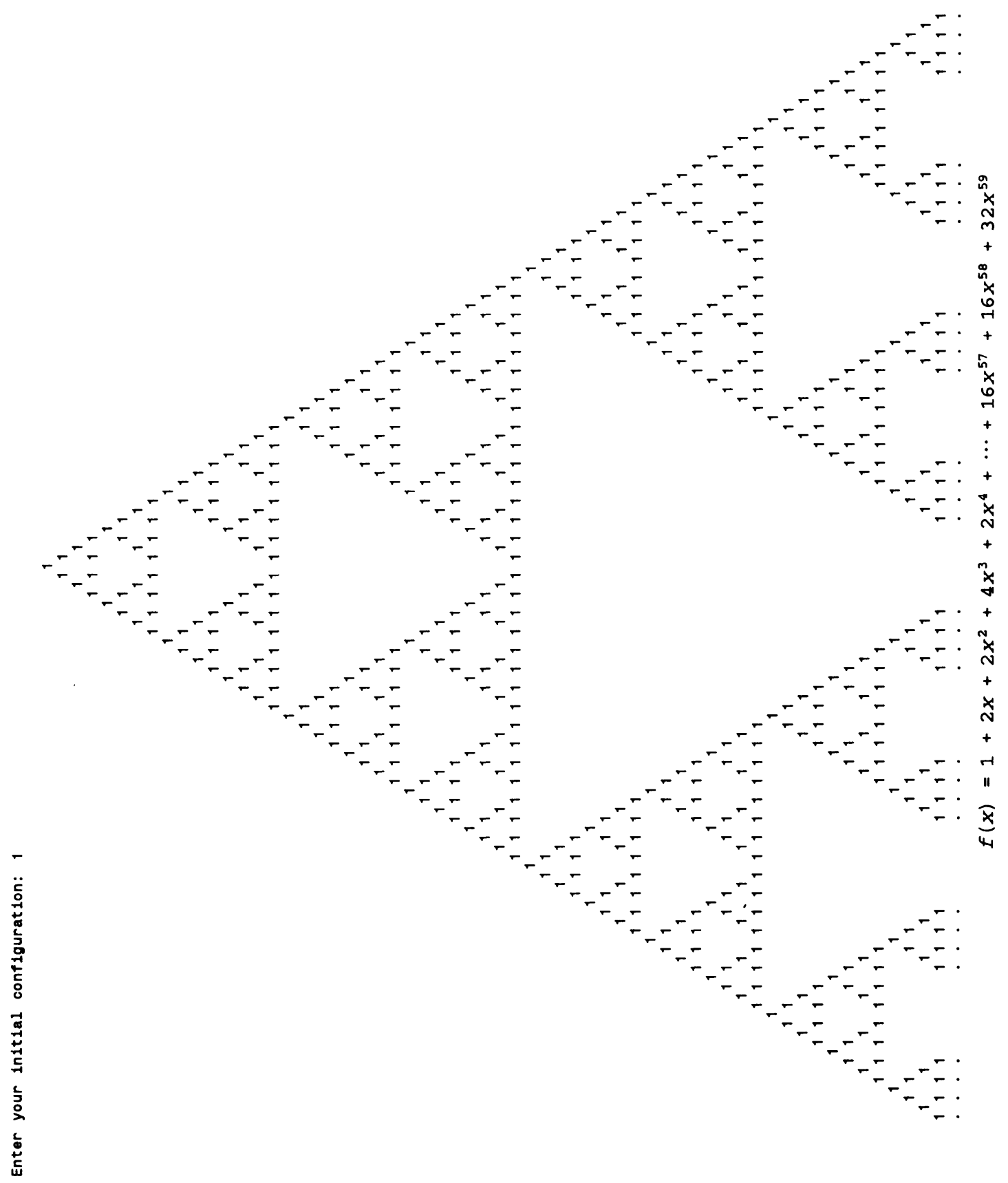

Fig. 1. The starting configuration has only one 1 . 


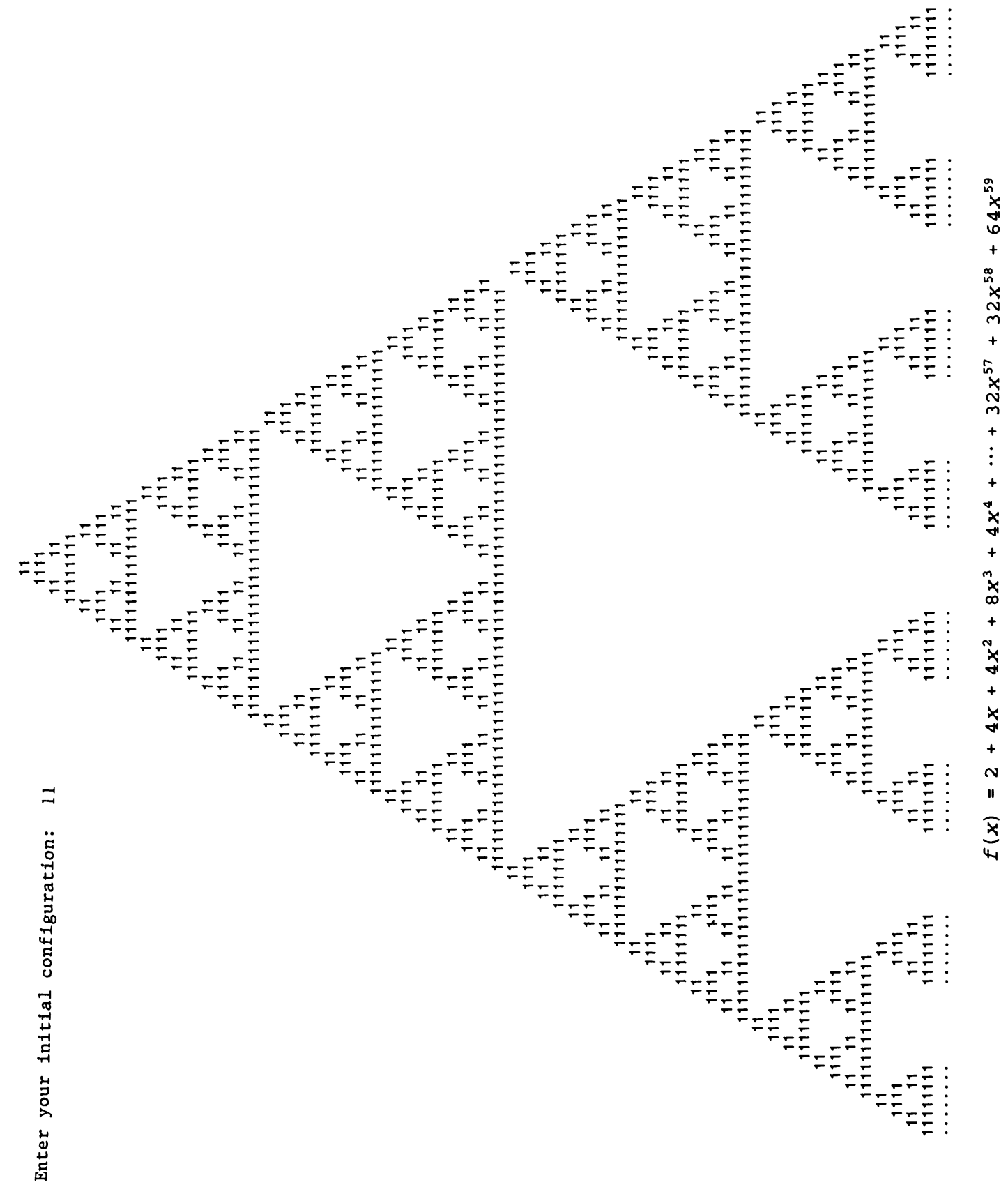

Fig. 2 The starting configuration is "11", that is, two 1's 


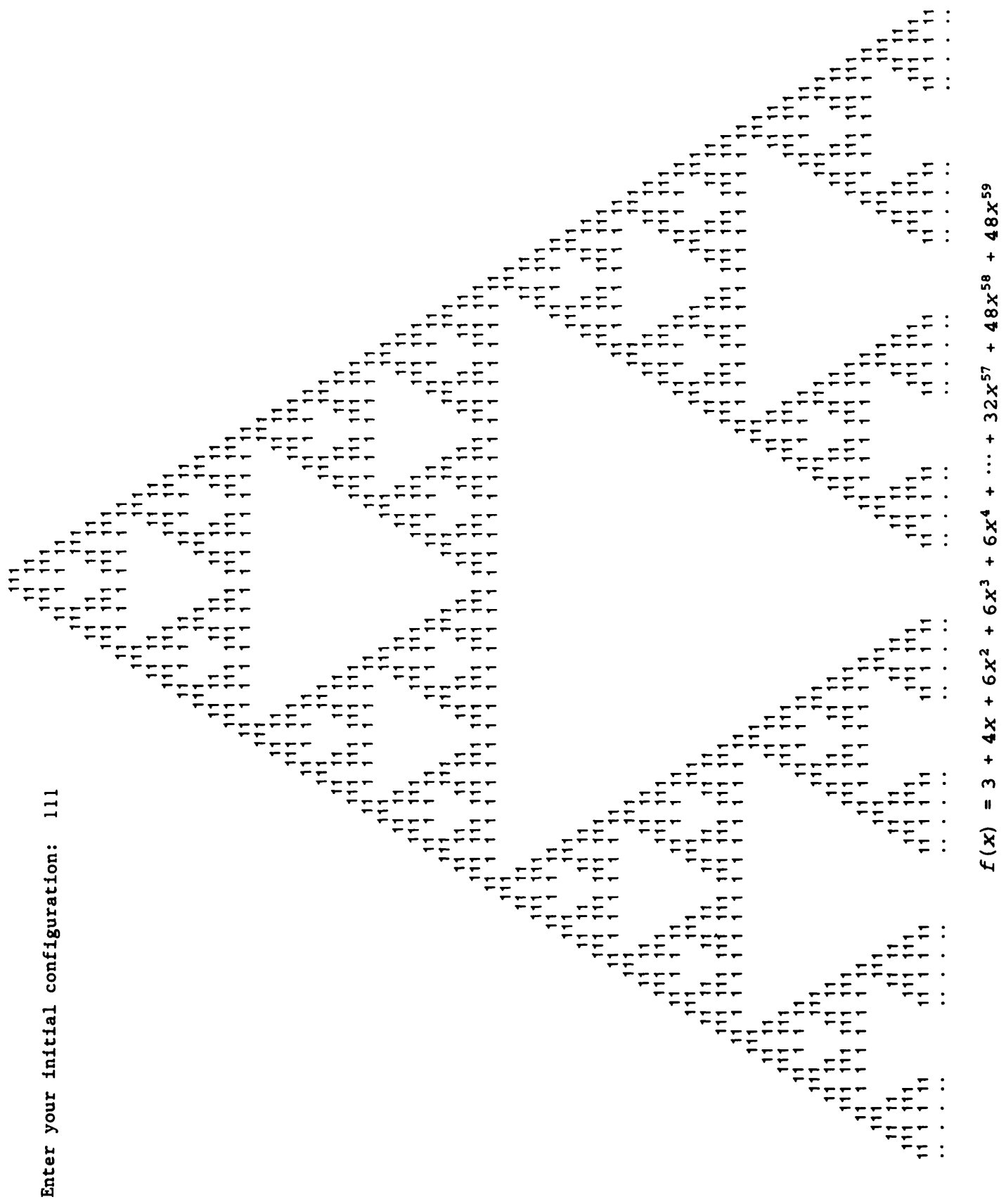

Fig. 3 The starting configuration is " 111 , that is, three 1 's" 


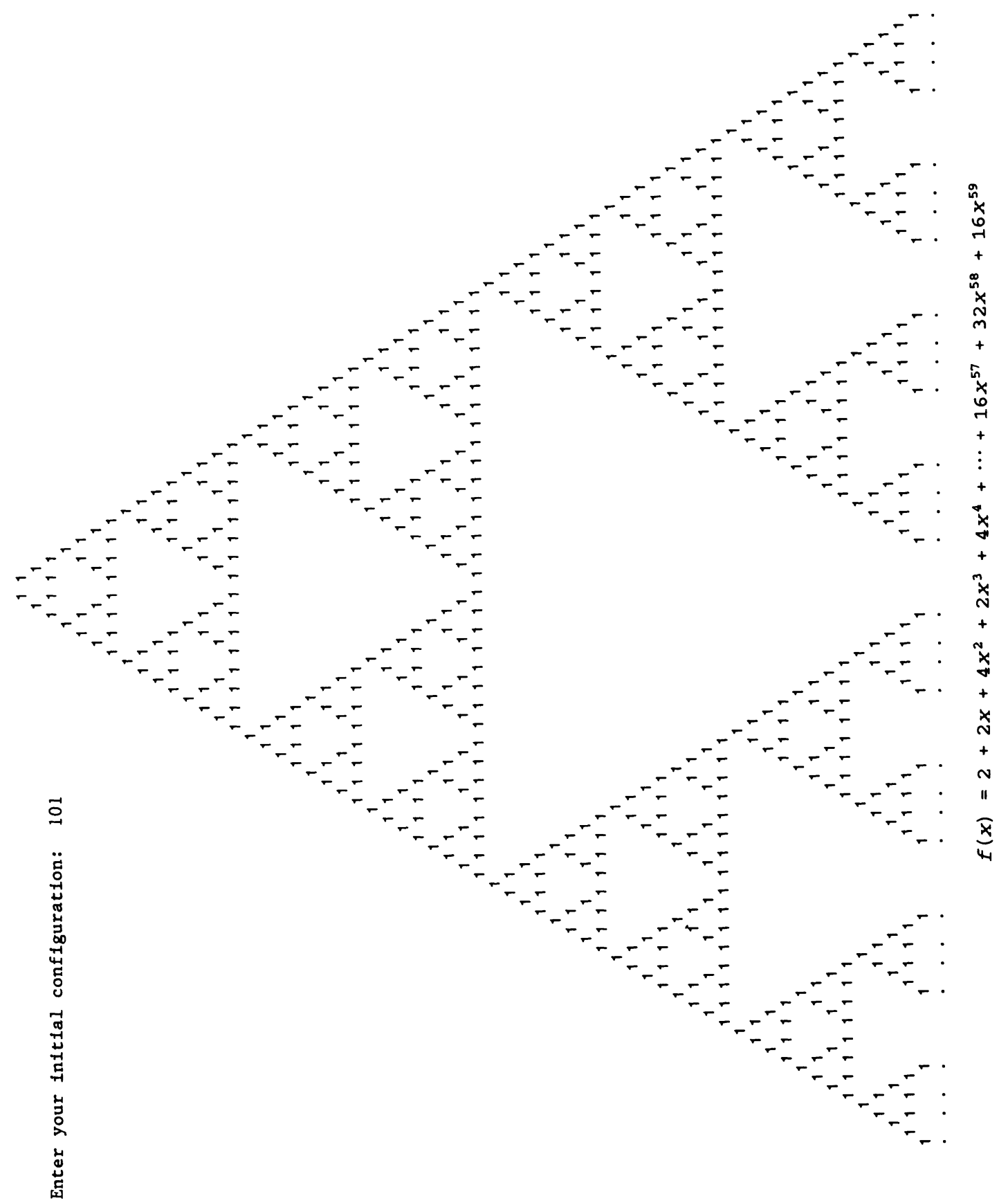

Fig. 4 The starting Configuration is "101, that is, one-zero-one" 
Because in Fig. 1, the triangle formed of stages 0 and 1 gives the first factor $1+2 x$. Then since it duplicates in the next stage (i.e., in Stage 3 ), and the corresponding term involving $x$ of the initial rov of stage 3 is $x^{2}$ (vhile considering the first triangle as a vhole, $i, e .$, equivalent to 1 ), then this yields to $1+2 x^{2}$, as the second factor of $f(x)$ in the factorized form, etc.

Similarly, the growth function in example 2 (see Fig. 2) can be factored as:

$$
g(x)=(2+4 x)\left(1+2 x^{2}\right)\left(1+2 x^{4}\right)\left(1+2 x^{8}\right)\left(1+2 x^{16}\right) \ldots
$$

Note that the factorization of $f$ and $g$ exhibit infinitely many zeros of the pover series which cluster at every point inside the unit circle in complex plane. So $f$ and $g$ must be non-rational.

In example 3 , the pattern is less regular. The "irregularity" first appears in the last row of stage 0 , and therefore ve can not factor the grovth function in this vay. Nevertheless, ve shall show that the associated grovth function of a general configuration-is alvays non-rational.

It is obvious that there is a natural relationship between an arbitrary starting configuration and the "fundamental configuration" since a general configuration is simply a "mode 2 " sum of shifts of the fundamental one. Indeed it follovs easily from the definition given below.

DEFINITION. If the starting configuration has $k$ number of 1 's and $I$ number of 0 's interspersed among them at the columns $j_{1}, j_{2}, \ldots, j_{l}$ then $i t s$ entries, $c_{i, j}$, can be obtained by the formula:

$$
\begin{gathered}
c_{i, j}=\sum_{t=0}^{k+l} d_{i, j-t}(\bmod 2) \\
t \neq j_{1}, \ldots, j_{l}
\end{gathered}
$$

where $k$ and $l$ are integers with $k \geq 1$ and $l \geq 0$.

Now, this definition including the fact that new stages on fundamental configuration begin at the rovs $2^{j}$ imply, no matter vhat the starting configuration is, the nev stages al rays start at the rovs $n=2^{j}$; and the corresponding terms of the grovth series are $a_{n} x^{2 j}$, where $a_{n}=2 a_{0}$.

THEOREY. In a One-dimensional Game of life, no matter vhat the starting configuration is, (finitely many 1 's and possibly sone 0 's in betreen), the associated grovth function is non-rational.

PROOF. Suppose, on the contrary, there is some case in vhich the associated grovth function

$$
f(x)=a_{0}+a_{1} x+a_{2} x^{2}+\cdots \cdot+a_{n} x^{n}+\cdots \cdot
$$

is rational. It is shown in [2] that if a pover series vith integral coefficients represents a rational function, then it can be expressed in the form $\frac{P(x)}{Q(x)}$ vhere $P(x), Q(x)$ are polynonials vith integral coefficients and $Q(0)=1$.

Therefore, if $f(x)$ is rational then there are polynonials $P(x)=\sum_{i=0}^{n} c_{i} x^{i}$, and $q(x)=\sum_{i=0}^{n} b_{i} x^{i}$, vhere $c_{i}, b_{i} \in Z$ and $q(0)=1$ (i.e., $b_{0}=1$ ) such that 
$f(x)=\frac{P(x)}{Q(x)}$

By doing simple algebra, this in turn imples that:

$$
\begin{aligned}
& a_{j}-c_{i}-\left(b_{1} a_{i-1}+b_{2} a_{i-2}+\ldots+b_{m} a_{i-m}\right) \text { for } i \leq n \text { and, } \\
& a_{i}=-\left(b_{1} a_{1-1}+b_{2} a_{i-2}+\ldots+b_{m} a_{i-m}\right) \text { for } i>n .
\end{aligned}
$$

(Sct $a_{i}-0$ for $j<0$. ) That is if $f$ is rational then there is a linear recurrence relation (2) for coefficients of the pover series after a certain number of terms. Assuming that such a linear recursion exists, there are integers $y_{1}, \ldots ., y_{m}$ such that:

$$
\mathrm{a}_{\mathrm{i}}=\mathrm{y}_{1} \mathrm{a}_{\mathrm{i}-1}+\mathrm{y}_{2} \mathrm{a}_{\mathrm{i}-2}+\cdots+\mathrm{y}_{\mathbf{m}} \mathrm{a}_{\mathrm{i}-\mathrm{m}} \text {, for } \mathrm{i}>\mathrm{n}\left(\mathrm{a}_{\mathrm{i}}=0 \text {, for } \mathrm{i}<0\right) \text {. }
$$

Now, we may choose $i$ large enough so that $i=q=2^{j}$, for some $j$ and $q>m$. Not. that rov q represents the first row of a nev stage. So, we have:

$$
\mathrm{a}_{\mathrm{q}}=2 \mathrm{a}_{0}, \mathrm{a}_{\mathrm{q}+1} \approx 2 \mathrm{a}_{1}, \ldots, \mathrm{a}_{\mathrm{q}+\mathrm{m}-1}=2 \mathrm{a}_{\mathrm{m}-1}, \mathrm{a}_{\mathrm{q}+\mathrm{m}}=2 \mathrm{a}_{\mathrm{m}} .
$$

From (3) and (4) we have:

$$
\begin{aligned}
a_{q+m+1} & =: y_{1} a_{q+m}+y_{2} q_{q+m-1}+\cdots+y_{m} a_{q+1} \\
& =2 a_{m} y_{1}+2 a_{m-1} y_{2}+\cdots+2 a_{1} y_{m} \\
& =2\left(\left(y_{1} a_{m}+y_{2} a_{m-1}+\cdots+y_{m} a_{1}\right) 4\right. \\
& =2 a_{m+1}
\end{aligned}
$$

Similarly

$$
\begin{aligned}
a_{q+m+2} & =y_{1} a_{q+m+1}+y_{2} a_{q+m}+\cdots \cdot y_{m^{a}}{ }_{q+2} \\
& =2 a_{m+1} y_{1}+2 a_{m} y_{2}+\cdots+2 a_{2} y_{m} \\
& =2\left(y_{1} a_{m+1}+y_{2} a_{m}+\cdots+y_{m} a_{2}\right) \\
& =2 a_{m+2} \\
& \\
\cdot & \\
a_{q+(q-1)} & =y_{1} a_{2 q-2}+y_{2} a_{2 q-3}+\cdots+y_{m} a_{2 q-m-1} \\
& =2 a_{q-2} y_{1}+2 a_{q-3} y_{2}+\cdots+2 a_{q-m-1} y_{m} \\
& =2 a_{q-1} .
\end{aligned}
$$

Also:

$$
\begin{aligned}
a_{2 q} & =y_{1} a_{2 q-1}+y_{2} a_{2 q-2}+\cdots+y_{m} q_{2 q-m} \\
& =y_{1}\left(2 a_{q-1}\right)+y_{2}\left(2 a_{q-2}\right)+\cdots+y_{m}\left(2 a_{q-m}\right) \\
& =2 a_{q} \\
& =2\left(2 a_{0}\right) \\
& =4 a_{0} .
\end{aligned}
$$


Therefore, ${ }^{a} 2 q={ }^{4 a} a_{0}$. But this is a contradiction, because rov $2 q=2\left(2^{j}\right)=2^{j+1}$ represents the first rov of a nev stage, i.e. Stage $(j+1)$, and hence $a_{2 q}=2 a_{0}$ and this completes the proof of the theorem.

\section{ACKNOYLEDGEMENT}

The author vould like to thank Dr. James Cannon for providing this problem and his valuable comments, $\mathrm{Dr}$. Donald Crove for revieving the final draft, and $\mathrm{Dr}$. Robert Knapp who has vritten the computer program.

\section{REFERENCES}

[1] Berlekanp, B. R. and Convay, R. K., Vinning Vays, Vol. II, Acadenic Press 1982, 817-850.

[2] Polya, G. and Szego", Problems and Theorens in Analysis, Vol. II, SpringerVerlag, Nev York and Heidelberg, Berlin, 1976. 138.

[3], Nolfram, Stephen, Geometry of Binomial Coefficients, Mmer. Mathe Monthly, Nov. 1984, 566-571. 


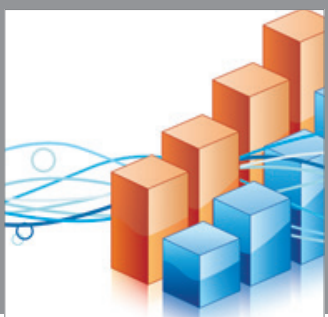

Advances in

Operations Research

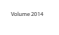

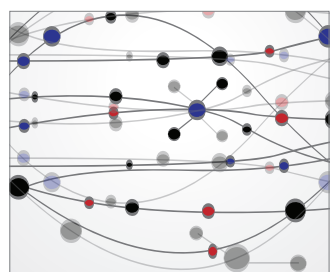

\section{The Scientific} World Journal
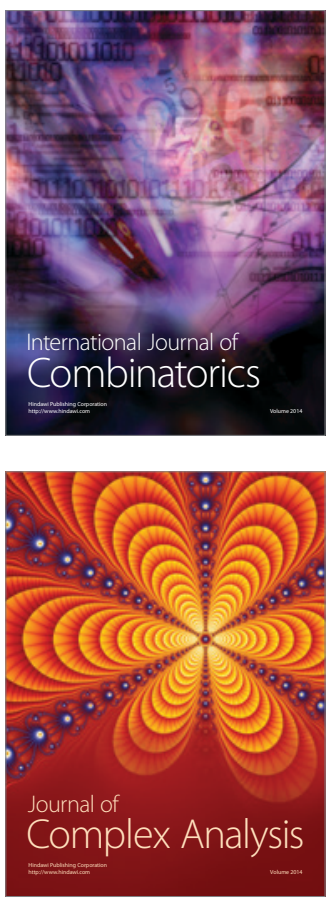

International Journal of

Mathematics and

Mathematical

Sciences
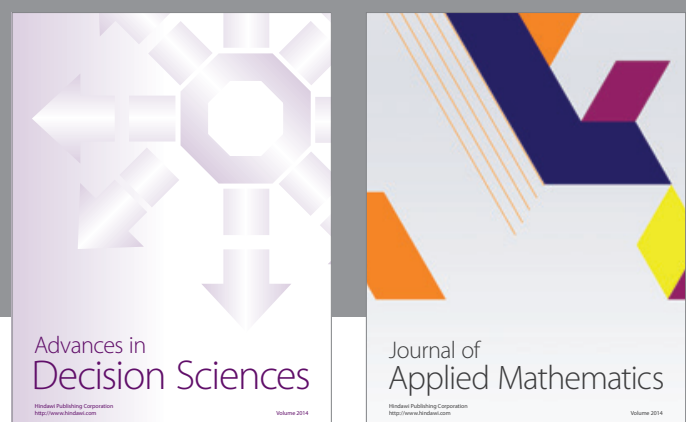

Journal of

Applied Mathematics
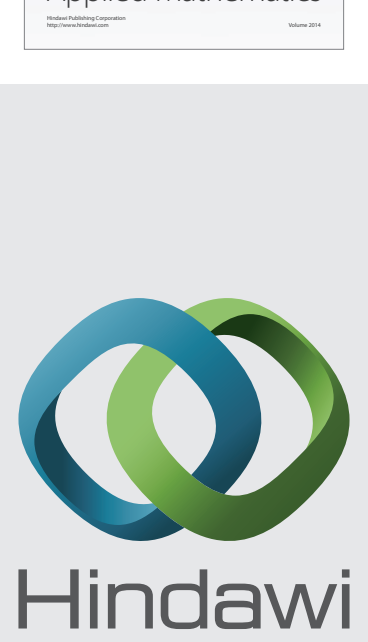

Submit your manuscripts at http://www.hindawi.com
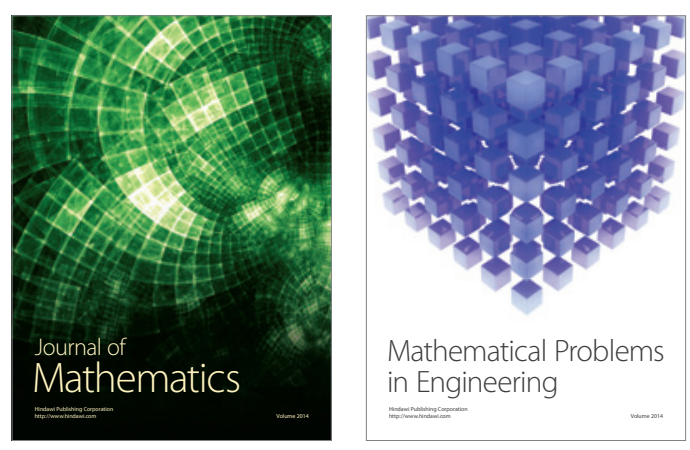

Mathematical Problems in Engineering
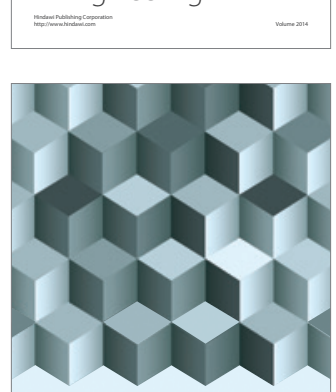

Journal of

Function Spaces
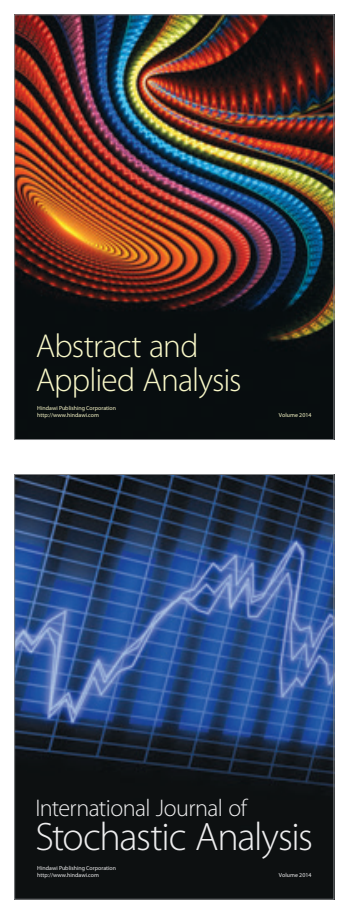

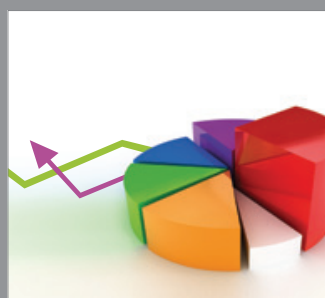

ournal of

Probability and Statistics

Promensencen
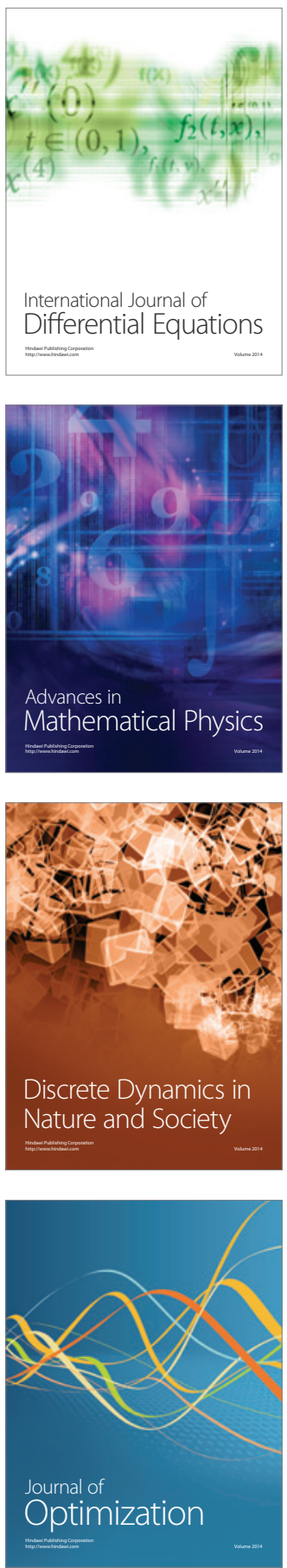